\title{
Schule und Raum. Inklusion und Exklusion als Prozessdimensionen sozialer Differenzierung
}

\section{Zusammenfassung}

Dieser Beitrag fokussiert zwei raumtheoretische Perspektiven: erstens die Betrachtung von Schule als Sozialraum innerhalb des umgebenden Sozialraums; zweitens die institutionelle und individuell-biografische Positionierung in Möglichkeitsräumen. Dabei kommen über die räumlichen Prozessdimensionen Inklusion und Exklusion Prozesse sozialer Differenzierung in den Blick.

Schlüsselwörter: Raum, symbolische Ordnung, Inklusion, Exklusion, Differenzierung

\section{School Space in the Field of Inclusion and Exclusion. Forming Order Through Differentiation}

\begin{abstract}
This paper focuses on two perspectives of spatial theory: first, the view of school as a social space within the environmental social space; second, the institutional and individual-biographical positioning in spaces of possibility. In doing so, an understanding of inclusion and exclusion as process dimensions of social differentiation will be developed. Keywords: space, symbolic order, inclusion, exclusion, differentiation
\end{abstract}

\section{Einleitung}

Der Raumbegriff in der Schulpädagogik wird in mindestens drei Varianten verwendet: manifest oder materiell, i.S.v. Schulgebäuden, Klassenzimmern und Pausenhöfen (Böhme \& Herrmann, 2009); metaphorisch, wenn von Bildungslandschaften, Lernoder Erfahrungsräumen die Rede ist (Liebau et al., 1999) und differenzierungstheoretisch, wenn es um räumliche (An-)Ordnungsstrukturen zwischen Inklusion und Exklusion geht (Hummrich, 2017). Die letztgenannte Perspektive soll hier im Zentrum stehen, auch wenn kursorisch auf die materielle Raumbedeutung fokussiert wird. Im Aufsatz werden zwei Schwerpunkte in den Blick genommen: erstens, die Untersuchung von Schule als Sozialraum in der sozialräumlichen Struktur ihrer Umgebung; 
zweitens die Perspektive auf den schulischen und individuell-biografischen Möglichkeitsraum. Abschießend werden zwei Forschungsperspektiven künftiger schulpädagogischer Raumforschung skizziert.

\section{Schule als Sozialraum}

Schulsysteme und gesetzliche Rahmungen zu Schule formulieren eine breite Inklusivität im Sinne einer umfassenden Teilhabeermöglichung. Hierin artikuliert sich die Beschreibung von Gesellschaften als „schooled societies“ (Baker, 2014), die mit dem Prinzip der Leistungsgerechtigkeit darauf zielt, die Chancengleichheit der Heranwachsenden sicherzustellen. Eine sozialraumanalytische Perspektive kann dazu beitragen, die Bedingungen schulischer Exklusivität in den Blick zu nehmen und die Verteilungskämpfe um schulische und soziale Privilegien differenziert zu untersuchen. Dabei soll (a) betrachtet werden, wie Schule als Sozialraum Bedingungen der Teilhabe jenseits der Leistungsgerechtigkeit schafft, und (b) welche Bedeutung die Position der Schule im Sozialraum der Umgebung (dem Viertel, dem Stadtteil, der Region) hat.

(a) Betrachtet man Schule als Sozialraum, wird die Perspektive auf die machtvolle symbolische Ordnung gerichtet. Dabei werden materiell-physische Räume als Ausdrucksgestalten dieser Ordnungen in den Blick genommen, die sich z. B. in Disziplinar- und Kontrollmechanismen der Schule artikulieren (Foucault, 1994). Den Zusammenhang von materiell-physischer Raumgestaltung und sozial-normativen Erwartungen analysieren Studien zur Schularchitektur (Böhme \& Herrmann 2009; Yamana, 1996) sowie zur Anordnung von Dingen am Ort Schule (Grabau \& RiegerLadich, 2014). Die normativen Erwartungen artikulieren sich strukturell auch in der Vorstellung disziplinierter Körper; die Einübung in eine dem Unterricht angemessene Lernhaltung: den Stift richtig zu halten, oder den Körper durch Tische und Stühle angemessen platzieren zu lernen, sind Beispiele hierfür (Alkemeyer, 2011; Pongratz, 2004).

In Unterrichtsanalysen zeigt sich zudem ein räumliches Prozessieren von Inklusion und Exklusion, bei dem es auch zu paradoxen Verstrickungen kommen kann. So sind in inklusiven Unterrichtsarrangements häufig exkludierende Elemente einbegriffen. In einigen Schulen z.B. sind separierte Räume - sogenannte „Differenzierungsräume“ Teil inklusiver Settings, die die Schüler*innen mit Förderdiagnose nach dem gemeinsamen Unterrichtsbeginn aufsuchen (Blasse et al., 2015); andere Schulen entsenden Schüler*innen, die „stören“, in sog. Trainingsräume, in denen sie ihr Handeln überdenken sollen oder individualisierte Aufgaben bekommen. Beide Formen sind damit nicht nur ein schulischer Kontrollmechanismus, sondern hier werden immanent Orte der Ausgrenzung geschaffen, die Inklusionsbestrebungen paradox erscheinen lassen (Budde \& Rißler, 2017). Schule als Sozialraum lässt sich so in der spezifischen Adres- 
sierung der Schüler*innen beschreiben (Hummrich, 2016). Sie greift auf gesellschaftliche Muster der Disziplinierung und Differenzierung zurück und reaktualisiert diese inkludierend oder exkludierend.

(b) Schule positioniert sich jeweils auch im Sozialraum: Sie adressiert nicht nur die Schüler ${ }^{\star}$ innen, sondern verweist zugleich auf Erwartungen in Bezug auf das Milieu und auf die Auseinandersetzungen mit der jeweiligen schulischen Umgebung (Hummrich, 2016). Auch diese Perspektive ist nicht essentialistisch zu verstehen, i. S. der Verortung in territorialen Bedingungen und deren Wirken auf Schule (Deinet \& Reutinger, 2004; Kessl et al., 2002). Vielmehr lässt sich hier die Verräumlichung sozialer Ungleichheit betrachten (Kessl \& Reutlinger, 2011). In einschlägigen Untersuchungen zeigt sich, wie sich die Bedingungen ungleicher Bildungschancen in unterschiedlichen Stadtteilen manifestieren und sich Dimensionen sozialer Segregation (z.B. ethnisch und sozialökonomisch) überlagern (Fölker et al., 2015; Kemper \& Weishaupt, 2015).

Die räumliche Ordnung kann sich aber auch dynamisch entwickeln. Ein Beispiel hierfür sind Gentrifizierungsprozesse, durch die Wohnviertel baulich und sozial aufgewertet werden. Frank-Olaf Radtke und Patricia Stošić (2008) zeigen in ihrer erziehungswissenschaftlichen Studie am Beispiel des Frankfurter Gallusviertels, dass in diesem marginalisierten Stadtteil die Zahl der Absolvent*innen mit „höheren“ Schulabschlüssen im Untersuchungszeitraum gering war, nicht zuletzt auch deshalb, weil im Stadtteil keine gymnasialen Schulformen vorhanden waren, wohl aber drei Förderschulen. In seiner aktuellen humangeografischen Studie zum gleichen Stadtteil zeigt Sebastian Schipper, dass durch den Ausbau des gehobenen Wohnsegments in den letzten Jahren kulturkapitalstarke und ökonomisch privilegierte Familien zunehmend die prekarisierten Familien verdrängen (Schipper, 2021). Gleichzeitig werden Schulneugründungen geplant, so dass die Attraktivität des Sozialraums für privilegierte Familien weiter steigt.

Diese Ambivalenzen (Ermöglichung von höherer Bildung und Verdrängung der prekarisierten Milieus) sind ein Hinweis darauf, dass Schule die Lernenden auf der Grundlage normativer Erwartungen adressiert, die (An-)Passung an die symbolische Ordnung der Schule bedeuten. Die Lernenden weisen jedoch herkunftsbedingte Unterschiede auf: Je nach Milieu und habitueller Orientierung treffen sie in der Schule auf bekannte und ihrer Herkunftsfamilie nahestehende oder ihnen befremdlich erscheinende Erwartungen. Kulturkapitalstarke und dem bildungsbürgerlichen Milieu nahestehende Kinder haben im Vergleich zu jenen aus wenig privilegierten Milieus deutliche Vorteile in der Anpassung an die symbolische Ordnung (Bourdieu \& Passeron, 1971). Die weniger privilegierten Schüler*innen werden schulisch nicht vollständig ausgegrenzt, sondern intern exkludiert: Sie verbleiben im System, aber die Teilhabe an exklusiven Bildungsarrangements wird ihnen verwehrt (Bourdieu, 1998). Befunde hierzu finden sich in jüngeren Untersuchungen von Schulen in nicht privi- 
legierten Milieus, z.B. in der Studie „Brennpunkt-Schule“ (Fölker et al., 2015). Gezeigt wird, dass schon die Bezeichnung der Schule als „Brennpunktschule“ durch Lehrer*innen eine immanente Abwertung der Schule darstellt und Anzeichen dafür ist, dass die Schüler*innen aus der Problem- und Defizitperspektive wahrgenommen werden. Ihnen wird, wie Stefan Wellgraf (2014) in seiner Studie „Schule der Gefühle" herausstellt, an diesem Ort vermittelt, dass sie minderwertig seien. Diese Studien zeigen, dass die Schüler*innen an diesem Ort zwar Teil des Systems Schule sind, ihnen aber Zugänge zu privilegierten Bildungsgängen verwehrt werden. Umgekehrt zeigt sich im Schulwahlverhalten von Mittelschichteltern, dass sie die Schule für ihre Kinder nach der Passung auswählen, was wiederum die begehrten Schulen veranlasst, sich in einer wettbewerblichen Struktur zu profilieren (Ackeren, 2006).

Schule im Sozialraum lässt sich als Institutionen-Milieu-Komplex beschreiben (Helsper \& Hummrich, 2008), in dem über Inklusion und Exklusion (häufig in Gestalt der internen Exklusion) Passungs- und Abstoßungsverhältnisse produziert werden. Dabei werden sozialräumliche Lagerungen der Herkunftsmilieus i.S. v. sozialen Differenzierungen reproduziert.

\section{Schule als Möglichkeitsraum der Teilhabe}

In der Darstellung der Perspektiven auf Schule als Sozialraum im Sozialraum (Hummrich \& Hinrichsen, 2022) wurde deutlich, wie dynamisch sich die Prozessierung von Inklusion und Exklusion gestaltet. Im Folgenden soll der raumanalytische Blick geweitet werden, indem Schule als Möglichkeitsraum beschrieben wird und so der „Ereignischarakter“ (Stichweh, 2016, S. 163) von Inklusion und Exklusion deutlich wird. Zunächst wird auf Inklusion und Exklusion als Prozessstrukturen des schulischen Möglichkeitsraums eingegangen. Anschließend werden Prozesse der sozialen Differenzierung in und durch Schule im intersektionalen Zusammenspiel betrachtet und anhand von Widerstandspraktiken Transformations- und Reproduktionsdynamiken der räumlichen Ordnung fokussiert.

\subsection{Inklusion und Exklusion im Möglichkeitsraum}

Die Vorstellung von Schule als Sozialraum im Sozialraum verweist implizit darauf, dass die Struktur des Raumes mit den Handlungsmöglichkeiten und -grenzen zusammenhängt. So ist die Raumstruktur einerseits als vorgängig zu verstehen, denn Räume haben eine Geschichte und sind dadurch historisch geworden; gleichzeitig kann die Struktur handelnd verändert werden. Das Zusammenspiel aus vorgefundener (vorgängiger) Raumstruktur und Veränderungspotenzialen untersucht Martina Löw (2008) beispielhaft. Die Autorin unterscheidet als räumliche Strukturierungsleistun- 
gen spacing und Syntheseleistung. Spacing ist markiert durch die Annahmen und Anordnungen, die einem Raum normativ zugrunde liegen. Die Syntheseleistung stellt die Verknüpfungsleistung dar, die Menschen über die Anordnungsstrukturen erbringen. Diese Strukturierungsleistung kann institutionell und individuell-biografisch im Spannungsfeld von Inklusion und Exklusion beschrieben werden:

(a) Die institutionellen Möglichkeiten als Eröffnung und Begrenzung der Bildungsteilhabe zeigen sich in der bereits benannten Untersuchung zu Bildungsräumen (Radtke \& Stošić, 2008): Die Absehung von der Möglichkeit einer Platzierung von Gymnasien in prekarisierten Stadtteilen lässt sich hier als „bildungspolitisches spacing" (Stošić, 2012, S. 18) bezeichnen, das mit Annahmen über die Leistungsfähigkeiten der Bewohner*innen eines Stadtteils verknüpft wird. Damit entsteht eine räumliche Anordnungsstruktur, in der Prekarisierung so lange fortgeschrieben wird, bis konkurrierende Ordnungsparameter (z.B. durch Wohnungsbau) Transformationsmöglichkeiten und -notwendigkeiten schaffen, sodass sich neue Strukturbedingungen der Teilhabe (s. o., Prozess der Gentrifizierung) und damit neue Syntheseleistungen ausbilden.

Die Perspektive auf Einzelschulen wurde weiter oben mit dem Institutionen-MilieuKomplex beschrieben (Helsper \& Hummrich, 2008). Hierzu liegen differenzierte schulkulturtheoretische Befunde vor, die zeigen, wie Schulen ihre Inklusivität und Exklusivität mit Blick auf das Bezugsmilieu und den Schülerinnenhabitus entwerfen (Helsper et al., 2018; Kramer, 2011; Thiersch, 2014). In der Perspektive des dynamisierten Möglichkeitsraum-Begriffs kann dies als einzelschulische Reaktion auf die Anordnungsstrukturen (spacing) und die schulischen Selbstentwürfe, die als Möglichkeit daraus gefolgert werden (Syntheseleistung), beschrieben werden.

(b) Individuell-biografisch werden die schulischen Orientierungen auf Inklusivität und Exklusivität in Teilhabe- und Zugehörigkeitsordnungen bedeutsam (Hummrich, 2016). Die Erfahrungen der (Nicht-)Zugehörigkeit gestalten sich als individuellbiografische Möglichkeitsräume aus (Hummrich et al., 2017, S. 283 f.). Alheit (2007) spricht davon, dass der „Rückgriff auf Handlungsressourcen, die aus frühen individuellen Positionserfahrungen stammen“, und „die Vorstellung von positionellen Chancen“ (ebd., S. 284) die individuelle Grundlage der Auseinandersetzung mit der gegenwärtigen und künftigen Positionierung in Möglichkeitsräumen seien. Auch normative Erwartungen an Teilhabe und (Selbst-)Ausschluss, wie sie etwa über Altersphasen gegeben ist, bedingen den Möglichkeitsraum. Bspw. impliziert die Jugendphase Erwartungen wie Autonomieentwicklung, Grenzüberschreitung und Generativität (Ecarius, 1997; King \& Koller, 2009), die sich als spacing fassen lassen. Jedoch ist es gerade die Auseinandersetzung mit eigenen Vorstellungen (Syntheseleistungen), die eine individuierende Transformationsdynamik ermöglicht. Diese ist mithin in gesellschaftliche Differenzverhältnisse eingelassen, aufgrund derer wiederum Inklusion und Exklusion prozessiert werden. 
Das Konzept des Möglichkeitsraums gestattet es, Inklusion und Exklusion auf unterschiedlichen Ebenen des Handelns (hier: institutionell und individuell-biografisch) dynamisch zu verstehen und damit die Doppelperspektive der vorgefundenen Struktur und ihrer handelnden Hervorbringung (Bourdieu, 1985) hinsichtlich institutioneller Teilhabebedingungen und biografischer Verortungsperspektiven weiterzudenken.

\subsection{Intersektionale Perspektiven auf Teilhabe}

Die Perspektive des Möglichkeitsraums bietet den Vorteil, dass nicht nur die Reproduktion von Inklusions- und Exklusionsmechanismen in den Blick genommen wird, sondern auch die Transformationsoptionen der Teilhabe analytisch zugänglich werden (z.B. Erhalt oder Aufstieg in der sozialräumlichen Ordnung). Bildungsteilhabe wird u. a. im Zusammenspiel der Kategorien Milieu, Gender, Ethnizität und Dis-/Ability betrachtet (Riegel, 2016). Sie sind für die Analyse räumlicher Ordnungen von Bedeutung, weil sie Einblick in die Hervorbringung exkludierender Ordnungsbildung geben. Meistens wird zunächst eine Differenzkategorie in den Vordergrund gestellt, von der aus die Relationierung zu weiteren Kategorien erfolgt. Dies wird auch in den folgenden Studien deutlich, anhand derer intersektionale Perspektiven auf Teilhabe im schulischen Möglichkeitsraum beschrieben werden.

(a) Mit der Studie „Institutionelle Diskriminierung“ (Gomolla \& Radtke, 2009) lässt sich aus raumanalytischer Perspektive das Zusammenspiel von Dis-/Ability, Milieu und Ethnizität fokussieren. Ausgehend von der Feststellung, dass im Bielefelder Landkreis die Schüler*innenzahlen zwar zurückgingen, aber die Zahl der Sonderschulen stabil blieb, ergab die Analyse der Daten, dass überproportional viele „Migrantenkinder" auf die Sonderschule überwiesen wurden. Proportional zum Rückgang der Schüler*innenzahlen insgesamt war ihre Zahl auf Sonderschulen angewachsen. Im Verlauf zeigte sich, dass häufig nicht Leistungsfähigkeit, sondern Milieuzugehörigkeit und Ethnizität Grundlage der Schulempfehlungen und der Konstruktion von Disability waren. Hierin wird ersichtlich, wie Leistung durch das Zusammenspiel sozialer Zuordnungen überlagert wird. Dies lässt sich als institutionelles spacing bezeichnen, in dem es aufgrund der Vorannahmen zu Syntheseleistungen kommt, die Exklusion i. S. v. sozialer Benachteiligung tradieren. Das Zusammenwirken von sozialem Raum, wie ihn etwa Pierre Bourdieu mit Fokus auf das Milieu konzipiert hat, und schulischem Möglichkeitsraum kann so verdeutlicht werden: Die „Migrantenkinder“ gehörten einem Arbeitermilieu an, von dem gleichzeitig angenommen wurde, es sei sowohl kulturell als auch milieubedingt „bildungsfern“. Insofern kann man hier von einem Wechselspiel aus (geringeren) Leistungserwartungen und Begrenzung der sozialen Zugehörigkeitskonstruktionen sprechen. Der schulisch exkludierende Möglichkeitsraum schränkt so die Teilhabe an privilegierenden Sozialraumstrukturen (durch Aufstieg in ein anderes Milieu) ein. 
(b) In Studien zu erwartungswidrigen Biografien steht hingegen der soziale Aufstieg trotz Mehrfachbenachteiligung im Fokus. So wurde in der Studie „Bildungserfolg und Migration“ (Hummrich, 2009) mit Blick auf Geschlechtszugehörigkeit, Ethnizität und Milieu herausgearbeitet, dass, entgegen der Erfahrung herabsetzender Zuschreibung der Institution, die Unterstützung durch signifikante Andere (Familie, einzelne Lehrer ${ }^{\star}$ innen, Freund- und Pflegschaften) eine bedeutsame Rolle bei der Realisierung von Bildungsteilhabe spielt. Anders als es die sozialräumlich benachteiligende Verortung und institutionelle Exklusionsbedrohung erwarten lassen, können durch signifikante Andere Handlungsressourcen generiert werden, die Teilhabe an privilegierenden und privilegierten Bildungsressourcen ermöglichen und somit in alternative Syntheseleistungen führen.

Verallgemeinernd zeigt sich: Sozialräumlich werden über Differenzkonstruktionen Annahmen zur Leistungsfähigkeit privilegierter und nicht privilegierter Menschen verknüpft (spacing). Dabei treffen Kinder und Jugendliche auf schulkulturelle Möglichkeitsräume, die Teilhabe über Ordnungsvorstellungen in spezifischer Weise regulieren (institutionelle Syntheseleistung). Den sozialräumlich und schulkulturell exkludierenden Strukturen kann individuell begegnet werden (individuelle Syntheseleistung). Dies ist jedoch abhängig von den Handlungsressourcen, die sich biografisch aufgeschichtet haben (Alheit, 2007). Dass Inklusivität hier von der individuellen Gestaltung des schulischen Möglichkeitsraumes abhängig ist, verweist schließlich darauf, dass sich strukturelle Dimensionen sozialer Ungleichheit nicht transformiert haben.

\subsection{Widerstand in der räumlichen Ordnung}

Die beschriebenen „Aufstiegsbiografien“ lassen sich auch als widerständige Anpassungen an den schulkulturellen Möglichkeitsraum beschreiben. Widerständig sind sie deshalb, weil die entsprechenden Personen sich gegen die institutionellen und gesellschaftlichen Exklusionsmechanismen wenden. Sie tun dies aber, indem sie sich an die Leistungsnormativität anpassen und so ihre Inklusion „erstreiten“. Vor dem Hintergrund dieser paradoxen Figur wird im Folgenden die räumliche Dimension von Widerstandspraktiken unter dem Fokus auf Inklusions- und Exklusionsstrukturen betrachtet. Auch hier wird exemplarisch auf zwei Studien Bezug genommen.

(a) Die raumanalytische Lektüre von Paul Willis' Untersuchung „Spaß am Widerstand“ (1979) demonstriert die Bedeutsamkeit der Widerstandspraxen männlicher Arbeiterjugendlicher hinsichtlich der Exklusivität des schulischen Möglichkeitsraumes (Löw, 2008). Während der institutionelle Möglichkeitsraum der Schule durch Gebäude, Schulzeiten und -stunden sowie Verhaltenserwartungen vorstrukturiert ist (spacing), widersetzt sich die Jungenclique aus dem Arbeitermilieu (Lads) dieser Teilhabeordnung. Sie schwänzen, entfernen sich vom Schulgelände, markieren ihren Widerstand durch Anrempeln anderer Personen oder herumliegende Zigarettenkippen. 
So kommt es zu widerstreitenden Synthetisierungen: Lehrer*innen synthetisieren Schule als institutionalisierte (An-)Ordnung von Wissen; die Lads synthetisieren die Schule als Ort des Widerstands (Löw, 2008, S. 118). Dabei bleibt jedoch die Raumkonstruktion der Lads flüchtig. Sie erfahren individuelle und kurzfristige Bestätigung, werden aber durch die institutionalisierte Synthetisierungsleistung der Schule von Bildungsprivilegien exkludiert. Dabei zeigt sich in dem Wechselspiel aus Sanktionierung und Widerstand insgesamt eine Stabilisierung des institutionellen Möglichkeitsraums und seiner (An-)Ordnungsstrukturen.

(b) Ina Herrmann nimmt in ihrer 2014 veröffentlichten Untersuchung „Vandalismus an Schulen. Bedeutungsstrukturen maskierender Raumpraktiken“ das Zusammenspiel des materiellen Schulraums, seiner architektonischen (An-)Ordnungsstrukturen und die Artikulationen von Widerstand durch Vandalismus in den Blick. Dazu analysiert sie die Aufbringung von Graffitis an Schulwänden. Auch dies ließe sich zunächst als (vergängliche) gegenkulturelle Synthetisierung lesen, da die Graffitis leicht zu entfernen wären. Gleichzeitig hebt sich das Graffiti von der Flüchtigkeit der Synthetisierungsleistung der Lads ab, da es eine deutlich wahrnehmbare Spur hinterlässt (ebd., S. 238).

Der Blick auf den dynamischen Möglichkeitsraum impliziert so die Chance, die raumbezogenen Aushandlungsprozesse um die (An-)Ordnungsstrukturen verstehend nachzuvollziehen. Das Beispiel des Widerstands zeigt die institutionalisierte Begrenztheit schulischer Inklusivität, aber auch dessen Bedeutung als Individuation ermöglichende Auseinandersetzung, in der die Möglichkeit eigener Einflussnahme auf die Raumstruktur erprobt werden kann.

\section{Resümee und Ausblick}

Mit dem Sozialraum können das Umfeld der Schule und die Adressierung der Schüler*innen für die Analyse zugänglich gemacht werden. Mit dem Möglichkeitsraum wird die dynamische Struktur des Raumes fokussiert. Den Perspektiven auf Schule als Sozialraum und Möglichkeitsraum ist gemeinsam, dass sie den schulischen Raum als Anordnungsstruktur verstehen, in der soziale Differenzierungen und gesellschaftliche Machtstrukturen reproduziert und verhandelt werden. Sozialräumlich werden Inklusion und Exklusion als Strukturdimensionen fokussiert und können über Begriffe wie Passung (und Abstoßung) als symbolische Raumordnungen in den Blick genommen werden. Die Perspektive auf den Möglichkeitsraum verweist auf das Prozessieren von Inklusion und Exklusion und damit auf die dynamische Auseinandersetzung mit institutionellen Grenzen und ihrer jeweiligen Verhandelbarkeit und Wandelbarkeit. 
Für künftige Forschungen zu Inklusion und Exklusion als Prozessdimensionen des schulischen Raumes ist nun die Frage bedeutsam, wie räumliche Anordnungsstrukturen erforscht werden können, wenn sich die Bedeutsamkeit bisheriger Raumkonstruktionen (scheinbar) auflösen. Dies betrifft zwei gesellschaftliche Entwicklungsdimensionen: (a) Transnationalisierung und (b) Digitalisierung.

(a) Prozesse der Transnationalisierung im Sinne von Grenzüberschreitung betreffen zunächst individuell-biografische Möglichkeitsräume: Von Transnationalisierung ist die Rede, wenn es um Migration und Flucht geht, aber auch wenn Fragen der Internationalisierung im Sinne der strategischen Karriereplanung bearbeitet werden. Beide Elemente spielen in Schule eine Rolle (Hummrich \& Pfaff, 2018), weisen jedoch unvereinbare Unterschiede auf: Während Migration und Flucht von Defizitannahmen begleitet ist, werden mit Internationalisierung Chancenstrukturen betrachtet. Zu fragen wäre, inwiefern sich hier die Schere hinsichtlich der Bildungszugänge öffnet, soziale Differenzen vertieft und gesellschaftliche Segregationsprozesse befördert werden. Zudem verweisen die hybriden Zugehörigkeitskonstruktionen in beiden Varianten darauf, dass die nationale Verfasstheit von Schule an ihre Grenzen gerät. Welche Bedeutung dies für institutionelle Möglichkeitsräume und deren Entwicklung hat, bleibt zu untersuchen.

(b) Digitalisierung - so auch der Eindruck in der Pandemie - scheint den physischen Raum als Ort zunächst unwichtig werden zu lassen. Zudem ist Digitalität längst allgegenwärtiger Teil individuell-biografischer Erfahrungen, sodass hier auch schon von Postdigitalität gesprochen wird (Jörissen et al., 2020). Diese Entwicklung geht einher mit der Reproduktion von Ungleichheitsverhältnissen (Keuchel \& Riske, 2020), die sich nicht nur in der Schlechterstellung prekarisierter Jugendlicher hinsichtlich ihrer technischen und materiellen Ausstattung zeigt, sondern auch in den jeweiligen Möglichkeiten, sich gewinnbringend auf digitale Bildungsinhalte $\mathrm{zu}$ beziehen (Jörissen et al., 2020). So ist anzunehmen, dass das Zusammenspiel von individuell-biografischem und institutionellem Möglichkeitsraum fortbesteht. Empirisch zu untersuchen ist das neuartige Zusammenspiel schulischen spacings und daraus abgeleiteter Syntheseleistungen mit Blick auf neue Teilhabemöglichkeiten und fortbestehende Exklusionsbedingungen.

\section{Literatur und Internetquellen}

Ackeren, I. van. (2006). Freie Wahl der Grundschule? Zur Aufhebung fester Schulbezirke und deren Folgen. Die Deutsche Schule, 98 (3), 301-310.

Alheit, P. (2007). Geschichten und Strukturen. Methodologische Überlegungen zur Narrativität. Zeitschrift für Qualitative Forschung, 8, 75-96.

Alkemeyer, T. (2011). Die Körperlichkeit des Lernens, der Bildung und der Subjektivierung. In I. Erler, V. Laimbauer \& M. Sertl (Hrsg.), Wie Bourdieu in die Schule kommt (S. 5568). Studienverlag. 
Baker, D. (2014). The Schooled Society. Stanford University Press. https://doi.org/10.1515/ 9780804790482

Blasse, N., Budde, J., Hinrichsen, M., Hummrich, M., Niemeyer-Jensen, B., \& Thon, C. (2015), Die Exklusivität des Inklusiven. In C. Siedenbiedel \& C. Theurer (Hrsg.), Grundlagen inklusiver Bildung, Teil 2 (S. 137-161). Prolog-Verlag. https://doi.org/ $10.2307 /$ j.ctvss $405 z .13$

Böhme, J., \& Herrmann, I. (2009). Schulraum und Schulkultur. In J. Böhme (Hrsg.), Schularchitektur im interdisziplinären Diskurs (S. 204-221). VS. https://doi.org/10.1007/9783-531-91868-6_13

Bourdieu, P. (1985). Sozialer Raum und Klassen. Suhrkamp.

Bourdieu, P. (1998). Das Elend der Welt. UVK.

Bourdieu, P., \& Passeron, J.-C. (1971). Die Illusion der Chancengleichheit. Klett.

Budde, K., \& Rißler, G. (2017). Raum und Räumlichkeit im pluralisierten Unterricht. Zeitschrift für Inklusion, 1 (4). https://www.inklusion-online.net/index.php/inklusion-on line/article/view/455

Deinet, U., \& Reutlinger, C. (Hrsg.). (2004). „Aneignung“ als Bildungskonzept der Sozialpädagogik. VS. https://doi.org/10.1007/978-3-322-80966-7

Ecarius, J. (1997). Lebenslanges Lernen und Disparitäten in sozialen Räumen. In J. Ecarius \& M. Löw, (Hrsg.), Raumbildung Bildungsräume (S. 33-62). Leske \& Budrich. https:// doi.org/10.1007/978-3-663-11236-5_3

Foucault, M. (1994). Überwachen und Strafen. Suhrkamp.

Fölker, L., Hertel, T., \& Pfaff, N. (Hrsg.). (2015). Brennpunkt(-)Schule: Zum Verhältnis von Schule, Bildung und urbaner Segregation. Barbara Budrich. https://doi.org/10.2307/j.ctvdf0dt8

Gomolla, M., \& Radtke, F.-O. (2009). Institutionelle Diskriminierung. Die Herstellung ethnischer Differenz in der Schule. VS. https://doi.org/10.1007/978-3-531-91577-7

Grabau, C., \& Rieger-Ladich, M. (2014). Schule als Disziplinierungs- und Machtraum. In J. Hagedorn (Hrsg.), Jugend, Schule und Identität (S.63-69). VS. https://doi. org/10.1007/978-3-658-03670-6_4

Helsper, W., Dreier, L, Gibson, A., Kotzyba, K., \& Niemann, M. (2018). Exklusive Gymnasien und ihre Schüler. VS. https://doi.org/10.1007/978-3-658-17080-6

Helsper, W., \& Hummrich, M. (2008). Arbeitsbündnis, Schulkultur und Milieu. In G. Breidenstein \& F. Schütze (Hrsg.), Paradoxien in der Reform der Schule (S. 43-72). VS. https://doi.org/10.1007/978-3-531-91053-6_3

Herrmann, I. (2014). Vandalismus an Schulen. Bedeutungsstrukturen maskierender Raumpraktiken. VS. https://doi.org/10.1007/978-3-531-19488-2

Hummrich, M. (2009). Bildungserfolg und Migration. Biografien junger Frauen in der Einwanderungsgesellschaft (2. Aufl.). VS. https://doi.org/10.1007/978-3-531-91744-3

Hummrich, M. (2016). Schule und Sozialraum. Erziehungswissenschaftliche Perspektiven. In A. El-Mafaalani \& P. Strohmeier (Hrsg.), Auf die Adresse kommt es an. Segregierte Stadtteile als Problem- und Möglichkeitsräume (S. 168-187). Beltz.

Hummrich, M. (2017). Inklusion und Exklusion. In J. Budde, M. Hietzge, A. Kraus \& C. Wulf (Hrsg.), Handbuch Schweigendes Wissen (S. 261-274). Beltz Juventa.

Hummrich, M., Hebenstreit, A., \& Hinrichsen, M. (2017). Möglichkeitsräume und Teilhabechancen in Bildungsprozessen. In I. Miethe, A. Tervooren \& N. Ricken (Hrsg.), Bildung und Teilhabe (S. 279-303). VS. https://doi.org/10.1007/978-3-658-13771-7_14

Hummrich, M., \& Hinrichsen, M. (i. E., 2022). Raumtheoretische Ansätze. In H.-H. Krüger, C. Grunert \& K. Ludwig (Hrsg.), Handbuch Kindheits- und Jugendforschung (3. Aufl.). VS. https://doi.org/10.1007/978-3-658-24801-7_10-1

Hummrich, M., \& Pfaff, N. (2018). Editorial, Transnationalisierung. Tertium Comparationis, 24 (2), 143-150. 
Jörissen, B., Schröder, M. K., \& Carnap, A. (2020). Postdigitale Jugendkultur. Kernergebnisse einer qualitativen Studie zu Transformationen ästhetischer und künstlerischer Praktiken. In S. Timm, J. Costa, C. Kühn \& A. Scheunpflug (Hrsg.), Kulturelle Bildung (S. 61-77). Waxmann.

Kemper, T., \& Weishaupt, H. (2015). Region und soziale Ungleichheit. In H. Reinders, H. Ditton, C. Gräsel \& B. Gniewosz (Hrsg.), Empirische Bildungsforschung (S. 245-257). VS. https://doi.org/10.1007/978-3-531-19994-8_18

Kessl, F., Otto, H.-U., \& Ziegler, H. (2002). Einschließen oder aufmachen? Der Raum, sein Kapital und seine Nutzer. In M. Riege \& H. Schubert (Hrsg.), Sozialraumanalyse. Grundlagen - Methoden - Praxis (2. Aufl.) (S. 191-206). VS. https://doi.org/10.1007/ 978-3-322-94995-0_10

Kessl, F., \& Reutlinger, C. (2011). Sozialraumorientierung in der Kinder-und Jugendhilfe. In H.-J. Dahme \& N. Wohlfahrt (Hrsg.), Handbuch Kommunale Sozialpolitik (S. 280-293). VS. https://doi.org/10.1007/978-3-531-92874-6_21

Keuchel, S., \& Riske, S. (2020). Postdigitale kulturelle Jugendwelten. Zentrale Ergebnisse der quantitativen Erhebung. In S. Timm, J. Costa, C. Kühn \& A. Scheunpflug (Hrsg.), Kulturelle Bildung (S. 79-96). Waxmann.

King, V., \& Koller, H.-C. (2009). Adoleszenz als Möglichkeitsraum für Bildungsprozesse unter Migrationsbedingungen. In V. King \& H.-C. Koller (Hrsg.), Adoleszenz - Migration - Bildung (2. Aufl.) (S. 9-26). VS. https://doi.org/10.1007/978-3-531-90332-3_1

Kramer, R.-T. (2011). Abschied von Bourdieu? VS. https://doi.org/10.1007/978-3-531-930688

Liebau, E., Müller-Kipp, G., \& Wulf, C. (1999). Metamorphosen des Raums. Deutscher Studienverlag.

Löw, M. (2008). Einführung in die Soziologie der Bildung und Erziehung (2. Aufl.). Barbara Budrich.

Pongratz, L. A. (2004). Freiwillige Selbstkontrolle. Schule zwischen Disziplinar- und Kontrollgesellschaft. In N. Ricken \& M. Rieger-Ladich (Hrsg.), Michel Foucault: Pädagogische Lektüren (S. 243-261). VS. https://doi. org/10.1007/978-3-322-85159-8_13

Radtke, F.-O., \& Stošić, P. (2008). ,Sozialraum ' und ,Netzwerke` - Semantiken kommunaler Integrationspolitik. In M. Bommes \& M. Krüger-Potratz (Hrsg.), Migrationsreport. Fakten - Analysen - Perspektiven (S. 77-112). Campus.

Riegel, C. (2016). Bildung - Intersektionalität - Othering. Transcript. https://doi.org/ $10.14361 / 9783839434581$

Schipper, S. (2021). Der Staat als Adressat städtischer sozialer Bewegungen. Wohnungspolitische Kämpfe und postneoliberale Konstellationen. Zeitschrift für Wirtschaftsgeographie, 65 (2), 58-71. https://doi.org/10.1515/zfw-2020-0027

Stichweh, R. (2016). Inklusion und Exklusion: Studien zur Gesellschaftstheorie. Transcript. https://doi.org/10.1515/9783839422946

Stošić, P. (2012). Lokale Bildungsräume zwischen Struktur und Handlung. In Tertium Comparationis, 18 (1), 12-24.

Thiersch, S. (2014). Bildungshabitus und Schulwahl. VS. https://doi.org/10.1007/978-3-65804170-0

Wellgraf, S. (2014). Verachtung. In J. Hagedorn (Hrsg.), Jugend, Schule und Identität (S. 317330). VS. https://doi.org/10.1007/978-3-658-03670-6_18

Willis, P. E. (1979). Spaß am Widerstand: Gegenkultur in der Arbeiterschule. Syndikat.

Yamana, Y. (1996). Die Struktur der „Übersichtlichkeit“ des Landerziehungsheims Haubinda. Zeitschrift für Pädagogik, 42, 408-421. 
Merle Hummrich, Prof. Dr. phil. habil., geb. 1970, Professorin für Erziehungswissenschaft mit dem Schwerpunkt Jugend und Schule, Fachbereich Erziehungswissenschaften, Goethe-Universität Frankfurt.

E-Mail: m.hummrich@em.uni-frankfurt.de

Korrespondenzadresse: Goethe-Universität Frankfurt am Main, Fachbereich Erziehungswissenschaften, Theodor-W.-Adorno-Platz 6, 60323 Frankfurt am Main 